\title{
Review of: "Desertification risk fuels spatial polarization in 'affected' and 'unaffected' landscapes in Italy"
}

Xinyuan Liang

Potential competing interests: The author(s) declared that no potential competing interests exist.

Desertification is one of the focuses of international research on land degradation, a process that disrupts the balance of naturally fragile ecosystems. Therefore, it is interesting to dynamically correlate the landscape patterns of desertification regions from landscape ecology. By dividing Italian land degradation vulnerability zones, this paper evaluates the evolution characteristics of several landscape-level indicators at the administrative division scale. It attempts to propose related regional action policies based on the research results. On the whole, the design ideas of the article are reasonable, the content is rich, and it has theoretical reference value. Nevertheless, some issues require further clarification by the authors.

1) Regarding selecting research units, why not consider watershed units? In my opinion, compared with administrative units, basin-scale studies are more conducive to a complete interpretation of ecosystemrelated issues.

2) What is the basis for selecting landscape indicators in Table 1? Why choose these 15 indicators? Is there collinearity between the different metrics?

3) Perhaps the author can add some land-use change processes in the overview of the study area to give readers a better understanding of the regional landscape characteristics in Italy since 1960.

4) At what scale (e.g. grid, area) is the ESA assessment performed? It is recommended that the authors supplement the data and sources involved in the ESA assessment in the form of a table in the main text or summarize the basic data of the ESA assessment mentioned in the article in the supplementary materials.

5) The weights of the four ESAI indicators are defined as 0.25 , which seems somewhat inappropriate. I do not think climate quality, soil, vegetation and land management are equally important in ESA assessments.

6) Supplement the basis for dividing the vulnerability interval $(1.225,1.375)$. It is mentioned in the article that the spatial resolution of the generated map is $1 \mathrm{~km} 2$. Is this the result that the resolution of all basic data is consistent? Again, authors need to supplement the data source description.

7) Table 3 shows the correlation coefficient between landscape indicators and vulnerability. Why not determine the correlation when selecting indicators to guide the selection of indicators? In addition, the correlation between the two is significantly different in different years, and it may not be meaningful to conduct follow-up analysis based on no significant correlation. How did the author deal with this problem? 8) The authors may supplement with a series of spatiotemporal maps, making the spatiotemporal changes 
in landscape index and vulnerability more specific to improve readability.

9) The font of Figure 2 is too small.

10) It is suggested to add specific suggestions to the discussion on how to control the action plan to prevent and control desertification based on the landscape process. 